\title{
Valoración del interés y conocimiento acerca de alimentación y actividad física en estudiantes del ciclo básico de la Facultad de Bioquímica y Ciencias Biológicas-UNL
}

Noseda J. • Cúneo F. • Fortino M.A. 


\section{Valoración del interés y conocimiento acerca de alimentación y actividad física en estudiantes del ciclo básico de la Facultad de Bioquímica y Ciencias Biológicas-UNL}

Noseda J. ${ }^{1}$, Cúneo F. ${ }^{2}$, Fortino M.A. ${ }^{1}$

afortino@fbcb.unl.edu.ar

${ }^{1}$ Cátedra de Qca Biológica, Dto de Cs Biológicas. Facultad de Bioquímica y Ciencias Biológicas-UNL. Ciudad Universitaria, Santa Fe, Santa Fe (3000), Argentina. +54 342575 211. Fax 0342 -4575221

${ }^{2}$ Cátedra de Trabajo Final de Licenciatura en Nutrición, Facultad de Bioquímica y Ciencias Biológicas-UNL. Ciudad Universitaria, Santa Fe, Santa Fe (3000), Argentina. +54 342575 216. Fax 0342 -4575221

\section{RESUMEN}

La alimentación se reconoce como un punto clave en el mantenimiento de la salud y la prevención de patologías. En jóvenes universitarios la educación alimentaria representa un desafío que no debe ser soslayado. Las Guías Alimentarias para la Población Argentina (GAPA), son una herramienta útil para favorecer la adquisición de estilos de vida saludables. El presente trabajo buscó identificar el interés y valorar el conocimiento sobre la alimentación (AL), la actividad física (AF), las GAPA y gráficas alimentarias en alumnos del ciclo básico de las carreras de Bioquímica (B) y las Licenciaturas en Biotecnología (LB) y Nutrición (LN) de la Facultad de Bioquímica y Ciencias Biológicas (FBCB)-UNL, a fin de evaluar la posibilidad de realizar intervenciones educativas futuras. Se encontró que entre los estudiantes predomina el interés acerca de AL y AF; que el conocimiento acerca de las GAPA y gráficas alimentarias es muy bajo para los alumnos de $B$ y $L B$, y que el hecho de conocer los mensajes principales de las GAPA contribuye a su práctica por parte de los estudiantes. Los resultados hallados ponen de manifiesto la necesidad de implementar propuestas educativas acerca de AL saludable y AF, principalmente en estudiantes de B y LB.

Palabras claves: universitarios; alimentación; actividad física; educación 


\section{SUMARY}

Evaluation of interest and knowledge about feeding and physical activity in students of the Department of Biochemistry and Biological Science-UNL.

Feeding is recognized as the key point in health maintenance and disease prevention. Food education represents a challenge in university students that should not be avoided. "Guías Alimentarias para la Población Argentina" (GAPA) are an useful tool to learn about how to get healthy habits for a healthy lifestyle. The aim of this research was to identify and value interest and knowledge about healthy feeding (HF) and physical activity (PA) in students from the three careers of Facultad de Bioquímica y Ciencias Biologicas-UNL; to assess the possibility of future educational interventions. It was found that interest for HF and PA predominate among the students; Biochemistry (B) and Degree in Biotechnology (DB) students have little knowledge about GAPA and food graphics; and the fact of knowing the main messages from GAPA helps to put them in practice. The results show that it's necessary to carry out a strong education programme to promote healthy habits among all the students.

Key Words: university students; physical activity; feeding; education.

\section{INTRODUCCIÓN}

La constitución de los hábitos alimentarios ocurre a partir de edades tempranas, pero los mismos pueden sufrir cambios que acompañan las nuevas situaciones de vida. Durante los años de universidad, los jóvenes suelen pasar muchas horas con actividades académicas que pueden provocar cambios alimentarios y de estilo de vida en general; con consecuencias negativas sobre la salud no solo en esta etapa de la vida sino también en etapas posteriores de la vida adulta (1-3). Varios estudios han revelado que la alimentación de los universitarios muchas veces no es la más adecuada, existiendo un alto consumo de tabaco y alcohol, y predominio de sedentarismo (4-6). En estudiantes de medicina de nuestra universidad se demostró que no escapan a esta realidad y que la dieta inadecuada resultó el factor de riesgo de obesidad y enfermedad cardiovascular de mayor prevalencia (7).

Las universidades son espacios en donde jóvenes y adultos pasan una parte importante de su vida trabajando y estudiando, por lo que resulta fundamental que se conviertan en sitios que promuevan la salud y los estilos de vida saludables (8).

Para favorecer una buena alimentación en las personas se necesita, entre otras cosas, de una educación alimentaria que brinde las herramientas necesarias para conocer qué es una alimentación sana y cómo elegir opciones de alimentos saludables. En nuestro país, las Guías Alimentarias para la Población Argentina (GAPA), son una herramienta disponible para realizar educación (9). Las primeras GAPA fueron propuestas en el año 2000 por la Asociación Argentina de Dietistas y Nutricionistas Dietistas (AADYND), y luego de más de 15 años 
fueron actualizadas y readaptas en función de los problemas nutricionales, epidemiológicos y de alimentación de la población resultando en las GAPA 2016.

El objetivo del presente trabajo fue valorar interés y conocimiento sobre alimentación saludable (AL) y actividad física (AF) en alumnos del ciclo básico de tres carreras de la Facultad de Bioquímica y Ciencias Biológicas de la Universidad Nacional del Litoral (UNL).

Este análisis pretende ser el punto de partida, reconociendo fortalezas y debilidades, para pensar estrategias en educación alimentaria nutricional que permitan a nuestros alumnos finalizar el ciclo básico con los conocimientos esenciales sobre esta temática en diferentes carreras.

\section{MATERIALES Y MÉTODOS}

Población: El estudio se llevó a cabo en dos grupos de estudiantes de ambos sexos, uno al inicio de la vida universitaria y otro al finalizar el ciclo básico, de las carreras de Bioquímica (B) y Licenciaturas en Biotecnología (LB) y Nutrición (LN) de la Facultad de Bioquímica y Ciencias Biológicas (FBCB). El trabajo se realizó en el período setiembre-noviembre de 2017, de tal modo que los ingresantes al menos contaban con un semestre de vida universitaria. Quedaron conformados los siguientes grupos:

Grupo 1 (G1): 225 alumnos que cursaban asignaturas del primer año de sus respectivas carreras. Para asegurar la representatividad de la muestra la cantidad de alumnos evaluados se calculó mediante la fórmula para poblaciones finitas (10), teniendo en cuenta la cantidad de ingresantes en 2017 a cada una de las carreras.

Grupo 2 (G2): 143 alumnos que constituían la totalidad de estudiantes que cursaban la asignatura Química Biológica ubicada en final del ciclo básico de los respectivos planes de estudio de las tres carreras (11).

Instrumento: Se utilizó una encuesta semiestructurada auto-administrada de 10 ítems (Figura 1), que se respondió en presencia de personal entrenado. En relación a los objetivos planteados, las preguntas 1 a 6 fueron seleccionadas a partir del cuestionario utilizado en la población adulta de USA por la Fundación del Concejo Internacional de Información Alimentaria (IFIC) en el proyecto Food \& Health Survey (12) y las preguntas 7 a 10 fueron elaboradas para el estudio. La validación de la encuesta se realizó mediante una prueba piloto y juicio de expertos en nutrición.

Los aspectos evaluados fueron: características generales de la población (edad, sexo, años de vida universitaria y carrera); interés acerca de AL, AF y peso corporal (P) y alimentación saludable (preguntas 1 y 4); conocimiento de las nuevas GAPA 2016 (preguntas 6, 9 y 10), y gráficas alimentarias de nuestro país y de otros países (pregunta 7).

Análisis estadístico: se utilizaron estadísticos descriptivos, media \pm desviación estándar (DE), frecuencias absolutas y porcentaje. 


\section{RESULTADOS Y DISCUSIÓN}

\section{Características de la población estudiada}

La tabla 1 resume las características de la población estudiada. LN fue la carrera más numerosa, ya que desde su creación supera la matrícula de ingreso de las otras dos. El predominio femenino encontrado es una constante de larga data en las tres carreras.

En relación a la edad de los alumnos, puede destacarse que si bien los promedios se ubican en 20 y 23 años ( $G 1$ y G2 respectivamente), los rangos se extienden a los 30 años para el inicio de la vida universitaria (G1) y aún más para su transcurso (G2). Si bien la cantidad de alumnos, cuyos años superan las edades promedio, no es numerosa (4\% para ambos grupos) constituye un factor más a tener en cuenta a la hora de contextualizar la realidad estudiantil universitaria.

Los años de vida universitaria del G2 indican el tiempo empleado en cumplimentar la currícula del ciclo básico en cada carrera, relacionados con los planes de estudio.

\section{Interés por alimentación, peso corporal y actividad física}

El interés del alumnado por alimentación, peso corporal y actividad física quedó demostrado a partir de las respuestas recogidas en la pregunta 1 del cuestionario. La opción alimentación fue seleccionada en $73 \%$ del G1 y $80 \%$ del G2; la opción $A F$ en $74 \%$ del G1 y $75 \%$ del G2 y la opción peso corporal por $49 \%$ del G1 y $55 \%$ del G2. El mayor porcentaje de alumnos encuestados manifestó estar interesado en las tres (AL, AF y P), seguido por el interés en AL y AF. Sólo un $6 \%$ del G1 y 3\% del G2 no demostró interés en ninguna de las propuestas (figura 2).

El interés por leer y/o escuchar acerca de alimentación y salud (pregunta 4) resulta sumamente importante a la hora de diseñar estrategias educativas. Las opciones en acuerdo (total y algo en acuerdo) sumaron $90 \%$ en el G1 ( $67 \%$ y $23 \%$ respectivamente) y $82 \%$ en el G2 (64\% y $18 \%$ respectivamente). Solo el $3 \%$ de los alumnos del G2 estuvieron en desacuerdo ( $1 \%$ algo y $2 \%$ total) con la afirmación 4 . Estos resultados se asemejan al estudio realizado en una universidad de Chile, donde los estudiantes también manifestaron su interés por la alimentación y la importancia que tiene la misma durante la etapa académica (13).

La información que intenta establecer conexiones entre alimentación y salud es un tema de candente actualidad y su abordaje es tan amplio como variado, utilizando la totalidad de los medios de comunicación ya sean tradicionales o nuevas tecnologías. Distintos estudios han coincidido que hoy en día el acceso a la información es casi irrestricto (14-16). Sin embargo, no toda la información disponible relacionada con la salud posee respaldo científico, por lo que se enfatiza la necesidad de contar con pautas claras para abordar esta temá-tica. Los resultados de la afirmación 5: “considero que la información sobre alimentación saludable es 
confusa o complicada" (figura 3), muestran que la información sobre alimentación saludable aún necesita ser revisada y se necesitan mayores esfuerzos para que los mensajes lleguen con claridad.

Con respecto a la valoración de su propia alimentación según la percepción del individuo (pregunta 2), la mayoría de los estudiantes en ambos grupos la calificó como medianamente saludable (figura 4). Cabe aclarar que estas respuestas se obtuvieron sin mediar ninguna referencia sobre lo considerado como alimentación saludable.

Un número importante de estudiantes de ambos grupos (65\% G1 y 63\% G2) afirmó haber realizado algún cambio en los últimos 6 meses buscando una alimentación más saludable (pregunta 3).

A diferencia de los estudiantes del presente trabajo, Troncoso y col. (17) entrevistaron universitarios chilenos y hallaron que la mayoría de ellos no percibía su alimentación como saludable. Ya revisando parámetros objetivos de la alimentación de universitarios, Saad Acosta y col. (18) revelaron que estudiantes de enfermería de una universidad de Colombia modificaron de manera negativa sus conductas alimentarias al ingresar a la facultad, por lo que su alimentación no era del todo saludable. Otro resultado similar fue encontrado por Cervera Burriel y col. (19) en estudiantes de una universidad de España, quienes presentaron un índice de alimentación saludable bastante bajo, dado que 91\% de los mismos manifestaban una "necesidad de cambio de su dieta".

\section{Conocimiento de las GAPA}

La existencia de las GAPA como documento oficial que promueve la alimentación saludable en nuestro país (pregunta 9) fue reconocido sólo por el $21 \%$ del G1 (79\% estudiantes de LN, $13 \%$ de B y $8 \%$ de LB) y $35 \%$ del G2 ( $90 \%$ alumnos de LN, $10 \%$ de B y ninguno de LB). La notable diferencia de este conocimiento en los alumnos de LN, podría estar asociada a un interés propio inherente a la elección de su carrera en el G1 (ingreso), y a que sólo estos estudiantes cuentan con asignaturas relacionadas a una alimentación saludable durante el ciclo básico (caso del G2).

Un estudio realizado en una universidad de Madrid valoró el conocimiento en materia de nutrición en alumnos de diferentes carreras y encontró que: los estudiantes de la Diplomatura en Nutrición Humana y Dietética presentaron conocimientos medios-altos, los alumnos de Farmacia y Enfermería conocimientos medios-bajos, y los alumnos de Podología mostraron una baja noción sobre la materia. Sin embargo no hubo diferencias significativas en hábitos alimentarios y estilo de vida entre las carreras (20).

Las GAPA 2016 cuentan con 10 mensajes principales, que establecen recomendaciones para una alimentación saludable y, a diferencia de las anteriores (GAPA 2000), hacen particular hincapié en la AF, incorporando la recomendación de realizar al menos 30 minutos diarios de AF. El mayor porcentaje de los alumnos de ambos grupos ( $49 \%$ G1 y $53 \%$ G2) afirmó que, de acuerdo a sus conocimientos, se deben practicar entre 
30-40 minutos al día de AF para promover una buena salud. La figura 5 muestra los resultados recogidos en la pregunta 6 .

Las GAPA son un instrumento educativo y, para poder pensar en estrategias efectivas de difusión del contenido (mensajes), es interesante reconocer qué saben los estudiantes acerca de los mismos. Partiendo de la idea que el conocimiento es fuente de empoderamiento para lograr la implementación de estilos de vida saludable en general, y de la alimentación en particular, se analizó el conocimiento de cada uno de los mensajes en las siguientes categorías: 1 . Conoce el mensaje; 2 . Conoce el mensaje y lo practica; 3 . Conoce el mensaje pero no lo practica. Bajo esta consigna, los resultados del ítem 10 de la encuesta se muestran en las figura 6.1 y 6.11.

Puede observarse que para la primera categoría (conoce) se encontraron valores superiores al $70 \%$ en G1 y al $80 \%$ en G2. En términos cualitativos, la tendencia en porcentaje de alumnos que conoce cada mensaje en G2 supera a G1, existiendo la posibilidad de que el conocimiento fuese adquirido en el tiempo transcurrido en la facultad. Otro aspecto a resaltar es la comparación de estos resultados de conocimiento de los mensajes con los resultados obtenidos en el conocimiento de las GAPA como documento (pregunta 9). En este sentido, la falta de identificación de los mensajes a partir de las GAPA, sugiere que fueron otros los medios por los que se informan acerca de estas recomendaciones. Estudiantes universitarios chilenos manifestaron que muchas veces la publicidad o su familia son quienes les informan acerca de conductas alimentarias saludables o de la importancia de realizar actividad física (13).

Con respecto a la práctica de los mensajes (conocimientos puestos en juego) se observa una notable disminución del porcentaje. Sin embargo, aparece el mismo patrón mencionado antes: hay una mayor tendencia al cumplimiento de cada mensaje en los estudiantes más avanzados (G2)

La variabilidad en el cumplimiento de los mensajes puede relacionarse con múltiples factores, de los cuales el conocimiento es solo uno de ellos, y en el presente estudio no se ha indagado sobre otras causas que hacen a la falta de práctica de un mensaje conocido. Varias investigaciones han encontrado que la falta de disponibilidad de tiempo, producto de las horas de estudios y de otras actividades académicas; como así también la situación económica, el entorno familiar o las preferencias alimentarias son algunos de los motivos mencionados por los universitarios para que la alimentación no sea la adecuada $(13,18,21)$.

Se ha comprobado que la educación alimentaria mejora por sí sola el comportamiento alimentario y el estado nutricional (22), siendo uno de los factores más importantes en todos los ámbitos para favorecer un estilo de vida saludable.

Rodríguez y col. (2) han encontrado que la falta de conocimientos acerca de alimentación en universitarios conduce a una mala lectura del rotulado nutricional contribuyendo a una mala elección de alimentos. En otro estudio, también con estudiantes universitarios, se identificó una asociación positiva entre el conocimiento de 
la ingesta diaria recomendada de calorías y el seguimiento en la recomendación del número de porciones de comida diaria (24).

El resultado encontrado para la categoría "no conoce el mensaje pero lo practica" muestra que entre quienes no conocen un mensaje, la práctica es extremadamente baja (frecuencia 3 a 6\% en G1 y 0 a 3\% en G2) y podría ser otro indicador de la importancia que tiene la educación para llevar un estilo de vida saludable.

\section{Conocimiento de las gráficas alimentarias}

Los lineamientos para una alimentación saludable son representados mediante gráficas, diferentes según el país. Seguramente, la gráfica más reconocida por la población general sea la pirámide nutricional, creada para la población estadounidense en los años 90, pero ampliamente difundida a nivel mundial por diferentes medios. El Óvalo Alimentario, fue la gráfica alimentaria desde el año 2000 hasta la publicación de las nuevas GAPA en el 2016, cuando fue reemplazado por el Plato Nutricional.

La identificación de las diferentes gráficas por los estudiantes se muestra en la figura 7. La pirámide fue la más conocida en ambos grupos (90\%). El óvalo, que por su período de difusión correspondería a la etapa de educación preuniversitaria de esta población, mostró la mayor diferencia entre G1 y G2; y el plato fue conocido por el $63 \%$ y $64 \%$ respectivamente. Todas las gráficas fueron mayoritariamente reconocidas por alumnos de LN. Solo un estudiante de LN (G1) y uno LB (G2) no reconocieron ninguna gráfica.

El 30\% del G1 y $45 \%$ del G2 fueron capaces de reconocer las 3 gráficas (pirámide, óvalo y plato), siendo los alumnos de LN los que representaron el mayor porcentaje (figura 8).

Al indagar acerca del lugar donde conocieron las gráficas señalaron en primer lugar la escuela (72\% G1 y $65 \%$ G2); mientras la universidad fue seleccionada por el $31 \%$ del G1 y $58 \%$ del G2.

Cuando se analizó la carrera de los estudiantes que señalaron la universidad como lugar de conocimiento de alguna de las gráficas se obtuvieron los siguientes resultados: LN 55\% del G1 y 95\% del G2, B 11\% del G1 y $24 \%$ del G2, y solo el $9 \%$ de los alumnos de LB en ambos grupos.

Estos resultados indican claramente la necesidad de implementar estrategias educativas destinadas a alumnos de LB y B que aborden esta temática. La carencia de información para mantener o llevar una alimentación saludable ha sido reconocida por otros estudiantes universitarios como uno de los factores condicionantes para llevar una dieta adecuada $(18,25)$.

\section{CONCLUSIONES}

La mayoría de los estudiantes manifestó interés por la AL y AF y, a pesar de que para muchos la información no les resulta demasiado clara, se percibe la intención de hacer su alimentación más saludable revelando que 
son jóvenes que están atentos a la temática. Sin embargo, la falta de conocimiento que presentan los estudiantes de B y LB resulta un factor desfavorable, evidenciando la necesidad de incluir educación alimentaria que puede ser satisfecha con diferentes formatos de propuestas educativas dentro de la facultad, con el fin de mejorar los estilos de vida de los estudiantes universitarios.

\section{AGRADECIMIENTOS}

El presente trabajo se realizó en el marco del Proyecto CAI+D 2016 (50120150100042LI): “Educación en Alimentación Saludable: una propuesta de docencia-investigación en estudiantes universitarios" financiado por la Universidad Nacional del Litoral.

Tabla1. Características generales de los estudiantes del grupo 1 y grupo 2.

\begin{tabular}{|c|c|c|c|c|c|c|}
\hline \multirow[b]{2}{*}{ Características Generales } & \multicolumn{2}{|c|}{ LN } & \multicolumn{2}{|c|}{ B } & \multicolumn{2}{|c|}{ LB } \\
\hline & Grupo 1 & Grupo 2 & Grupo 1 & Grupo 2 & Grupo 1 & Grupo 2 \\
\hline Alumnos FA* & 108 & 74 & 70 & 46 & 47 & 23 \\
\hline Alumnos $\mathrm{FR} \%{ }^{* *}$ & 48 & 52 & 31 & 32 & 21 & 16 \\
\hline Edad en años (media \pm DE) & $20 \pm 2.0$ & $21 \pm 2.3$ & $20 \pm 1.8$ & $24 \pm 4.6$ & $20 \pm 1.6$ & $22 \pm 2.7$ \\
\hline Rango en años & $18-30$ & $19-30$ & $18-28$ & $20-44$ & $18-27$ & $20-29$ \\
\hline Género(FA): F/M/NC ${ }^{* * *}$ & $89 / 19$ & $66 / 8$ & $52 / 16 / 2$ & $31 / 15$ & $30 / 17$ & $17 / 6$ \\
\hline Sexo (FR\%):F/M/NC & $82 / 18$ & $89 / 11$ & $74 / 23 / 3$ & $67 / 33$ & $64 / 36$ & $74 / 26$ \\
\hline $\begin{array}{c}\text { Años universitarios } \\
\text { (media } \pm D E \text { ) }\end{array}$ & $1 \pm 1.1$ & $3 \pm 1.2$ & $1 \pm 1.2$ & $5 \pm 2.1$ & $1 \pm 1.1$ & $5 \pm 2.3$ \\
\hline
\end{tabular}

${ }^{\star}$ FA: Frecuencia Absoluta; ${ }^{* \star}$ FR\%: Frecuencia Relativa en \%; ${ }^{* * *}$ NC: No contestó, F: femenino, M: masculino. 
ENCUESTA DE CONOCIMIENTO SOBRE ALIMENTACION Y SALUD

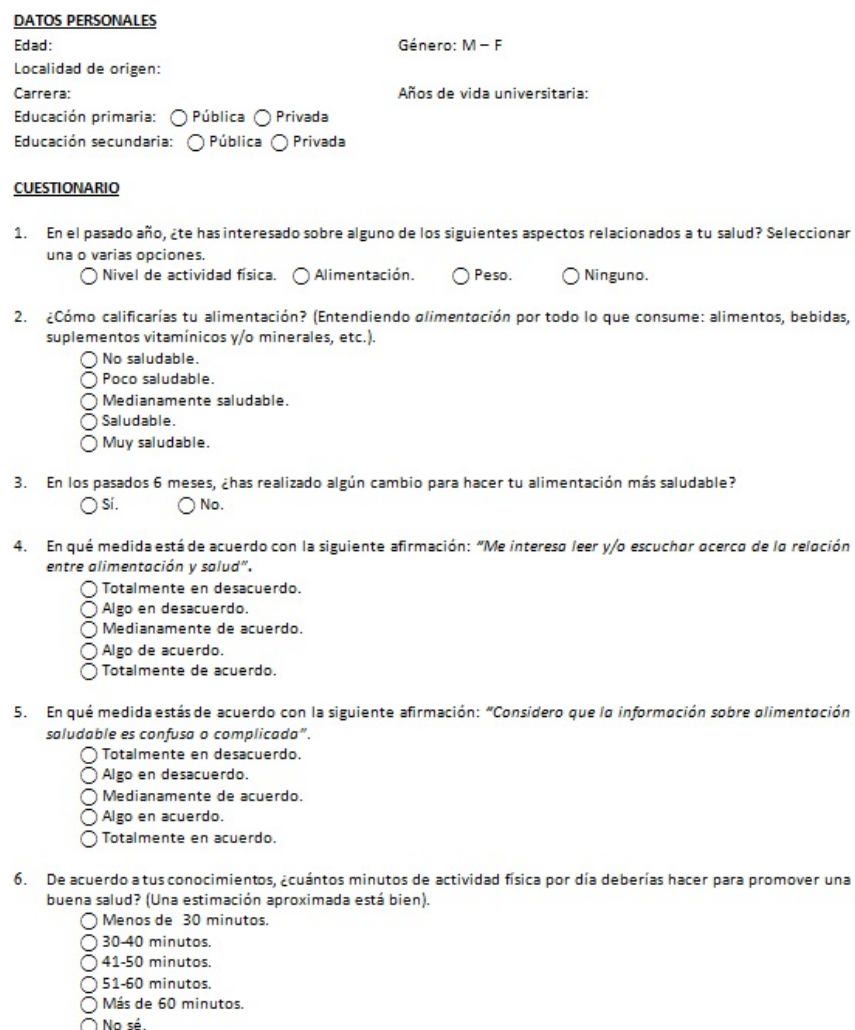

6. De acuerdo a tus conocimientos, ecuántos minutos de actividad fisica por día deberías hacer para promover una buena salud? (Una estimación aproximada está bien).

Menos de 30 minutos.

30-40 minutos.

$41-50$ minutos.

$51-60$ minutos.

Más de 60 minutos.

Figura 1.Encuesta utilizada.

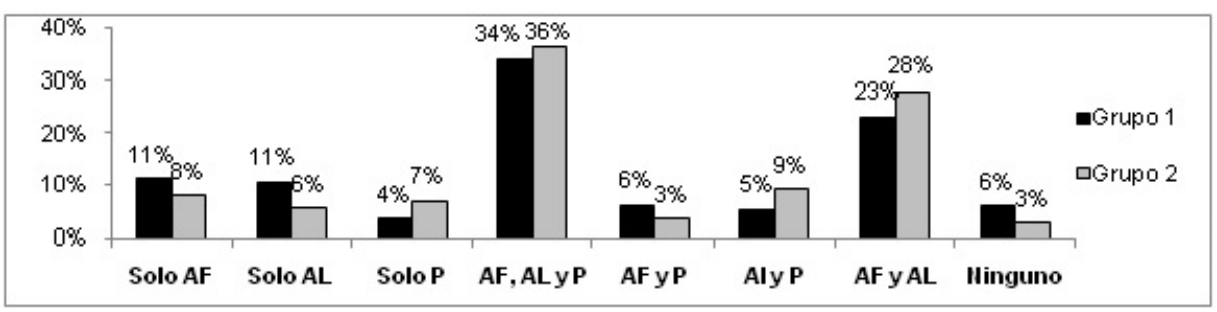

Figura 2. Alumnos que se interesan por alimentación (AL), actividad física (AF) y/o peso (P). 


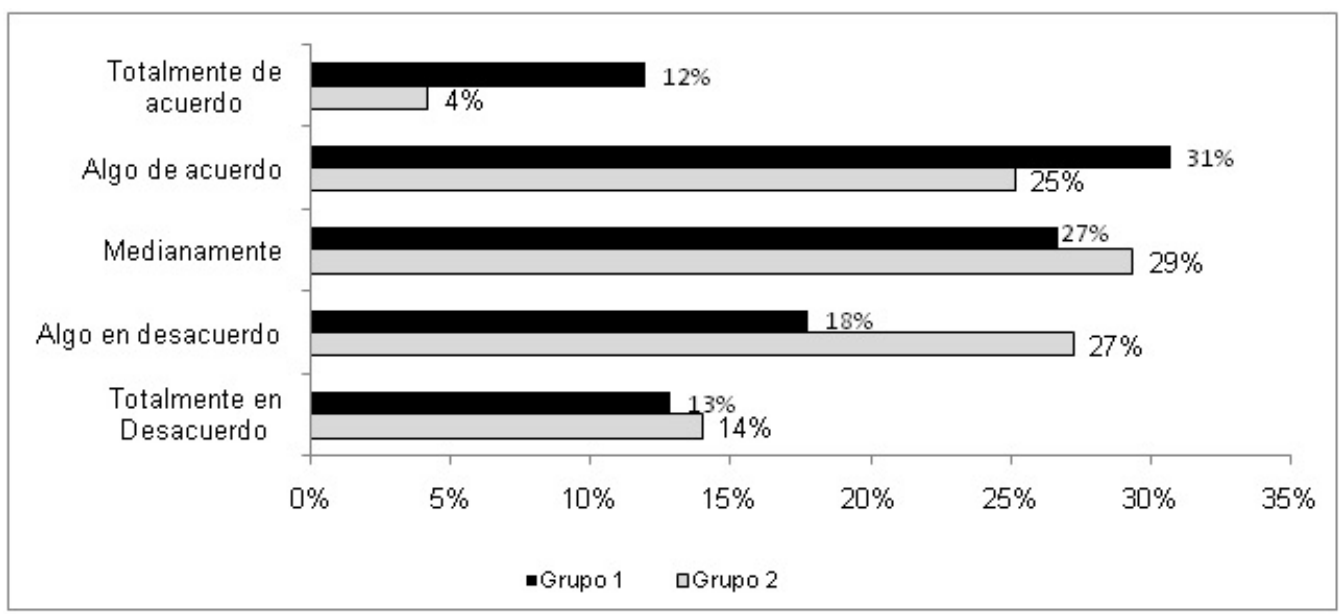

Figura 3. Respuesta de la afirmación 5 : "considero que la información sobre alimentación saludable es confusa o complicada" para ambos grupos.

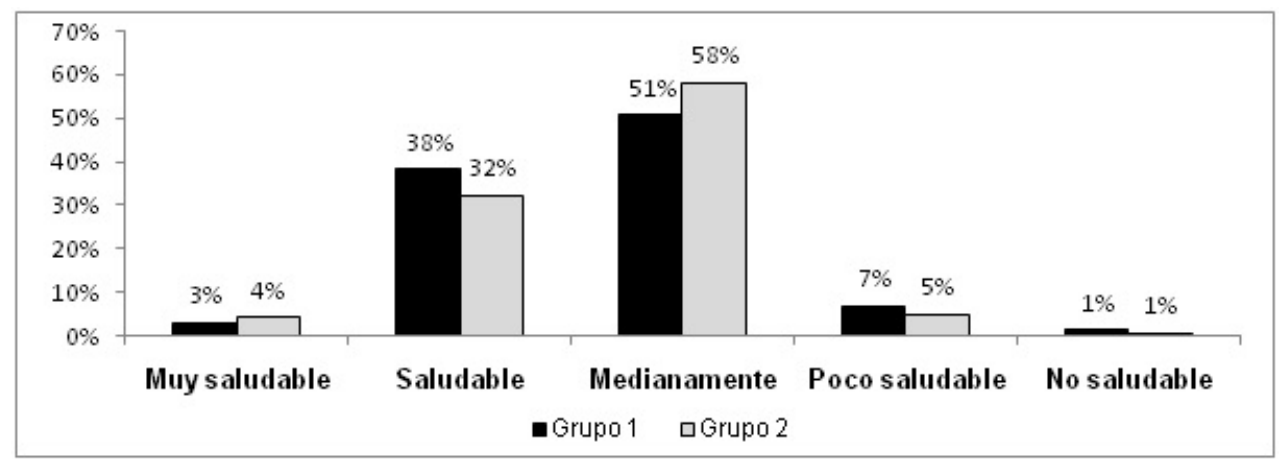

Figura 4. Valoración de la alimentación de los estudiantes de ambos grupos.

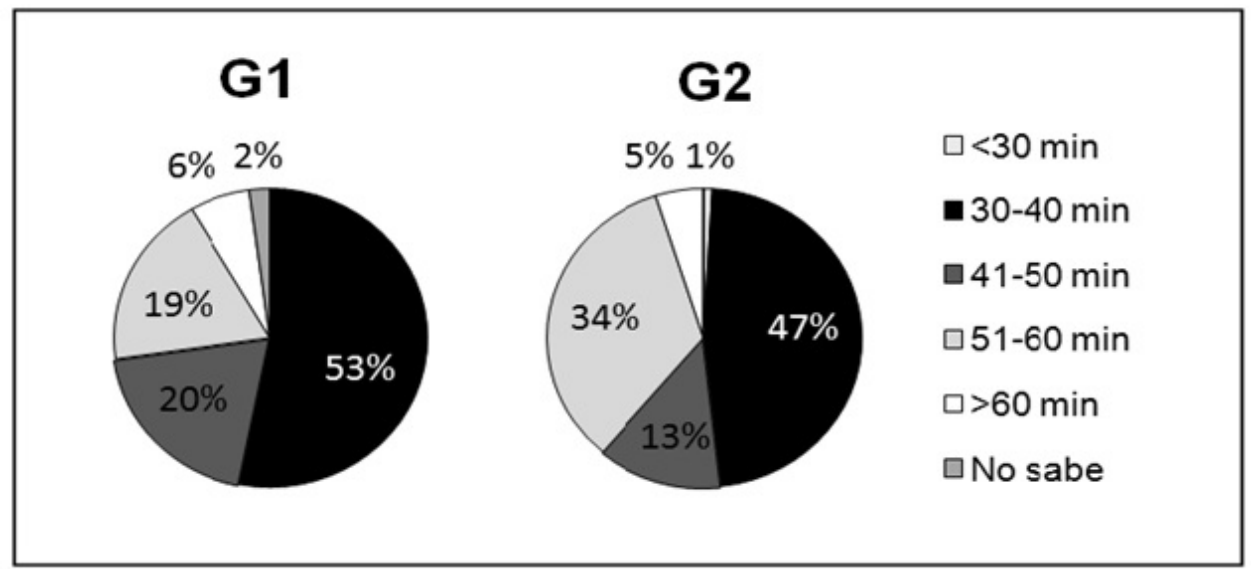

Figura 5. Tiempo de AF que los estudiantes de G1 y G2 creen que deberían hacer para promover una buena salud. 


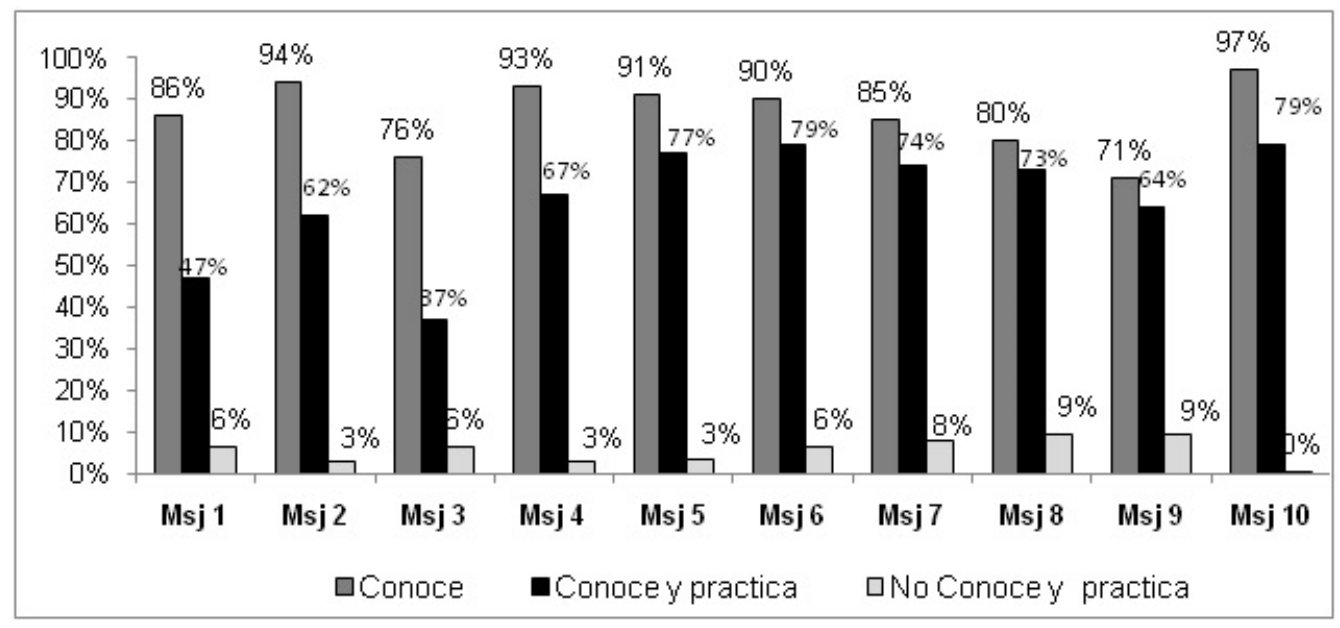

Figura 6.I. Porcentaje de alumnos del G1 que conoce, conoce y practica o conoce y no practica los mensajes (Msj) de las GAPA.

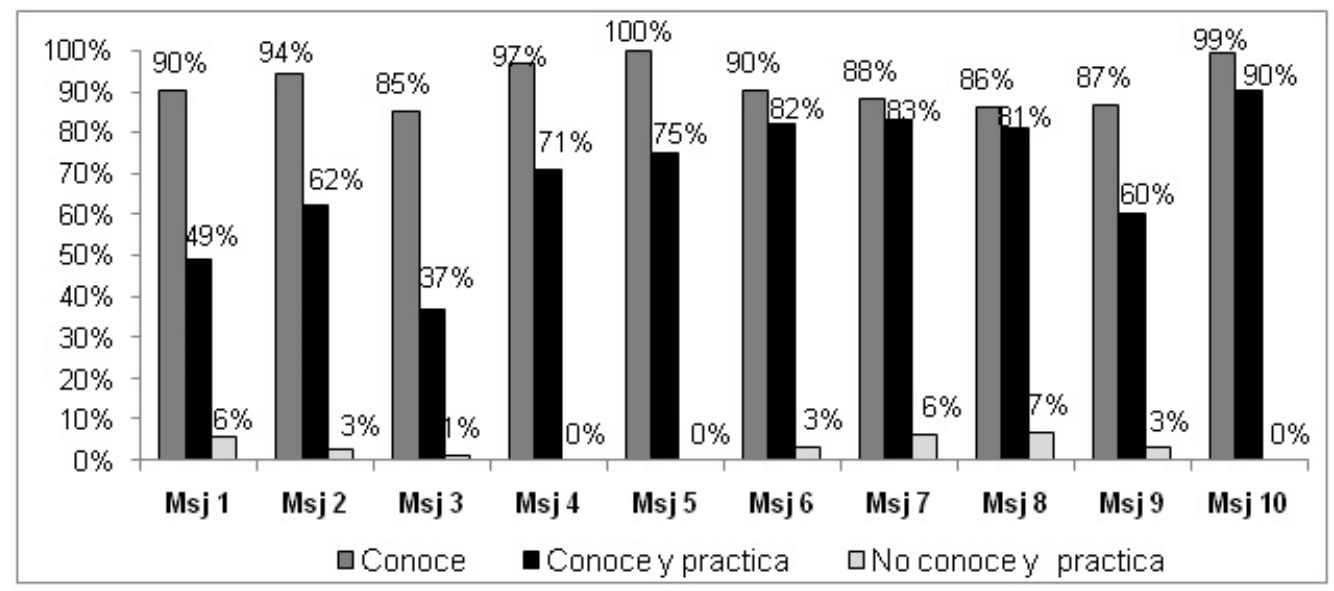

Figura 6.II. Porcentaje de alumnos del G2 que conoce, conoce y practica o conoce y no practica los mensajes (Msj) de las GAPA.

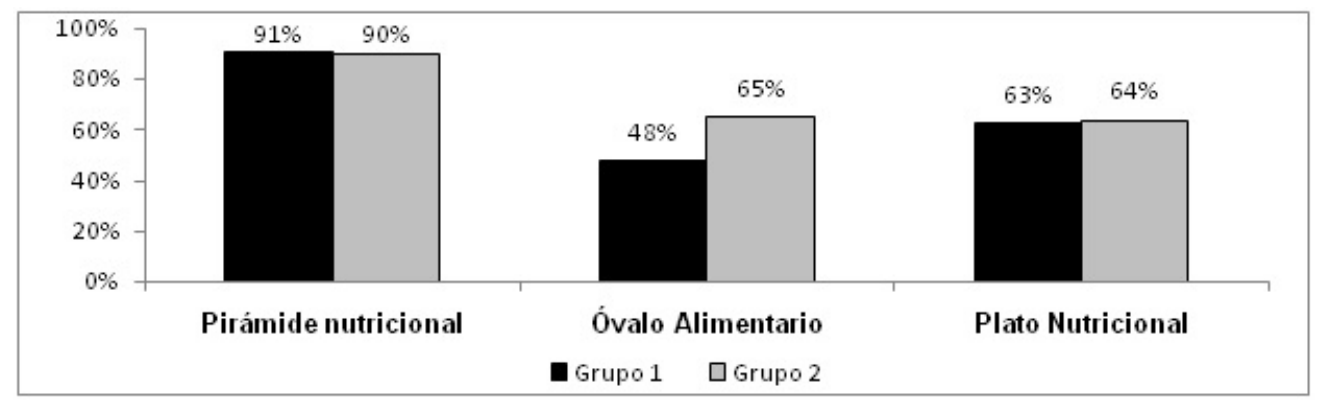

Figura 7. Alumnos de ambos grupos que conocen cada gráfica (Pirámide Nutricional, Óvalo alimentario y Plato Nutricional) 


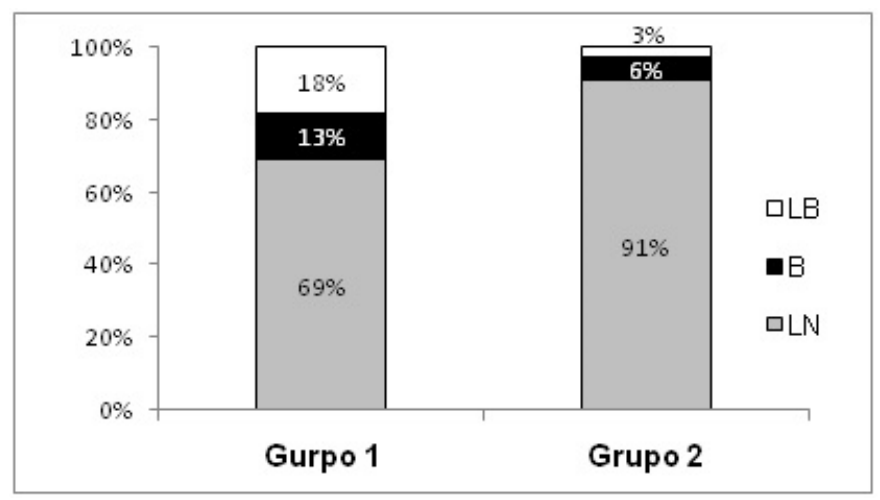

Figura 8. Distribución por carreras de los alumnos que conocen las 3 gráficas para los grupos 1 y 2 .

\section{BIBLIOGRAFÍA}

1. Irazusta Astiazaran A, Hoyar Cillero I, Díaz Ereño E et al. (2007). Alimentos de estudiantes universitarios. Osasunaz. 8: 7-18.

2. Ledo-Varela M.T., De Luis Román D.A., González-Sagrado M. y col., 2011. Características nutricionales y estilo de vida en universitarios. Nutrición Hospitalaria. 26, 4: 814-818.

3. MacMillan N.K., 2007. Valoración de hábitos de alimentación, actividad física y condición nutricional en estudiantes de la Pontificia Universidad Católica de Valparaíso. Revista chilena de Nutrición, 34, 4: 330336.

4. Espinoza L.O., Rodríguez F.R., Gálvez J.C. y col., 2011. Hábitos de alimentación y actividad física en estudiantes universitarios. Revista chilena de Nutrición. 38, 4: 458-465.

5. Martínez Roldán C., Veiga Herreros P., López de Andrés A. y col., 2005. Evaluación del estado nutricional de un grupo de estudiantes universitarios mediante parámetros dietéticos y de composición corporal. Nutrición Hospitalaria. 20, 3: 197-203.

6. Oviedo G., Morón de Salim A., Santos I. y col., 2008. Factores de riesgo de enfermedades crónicas no transmisibles en estudiantes de la carrera de Medicina. Universidad de Carabobo, Venezuela. Año 2006. Nutrición Hospitalaria. 23, 3: 288-293.

7. Monsted C., Lazzarino M.S, Modini L.B. y col., 2014. Evaluación antropométrica, ingesta dietética y nivel de actividad física en estudiantes de medicina de Santa Fe (Argentina). Rev. española de nutrición. 18, 1: 3-11.

8. Ministerio de Salud de la República Argentina (MSAL), 2012. Manual de universidades saludables. Buenos Aires. 50p.

9. Ministerio de Salud de la República Argentina (MSAL), 2016. Guías Alimentarias para la Población Argentina (GAPA). Buenos Aires. Documento Técnico Metodológico: 257p. 
10. Ronald E. Walpole, 2012. Probabilidad y Estadística para Ingeniería y Ciencias, Pearson Educacion (México) IX. 1-792.

11. Planes de estudio de las carreras de Bioquímica, Licenciatura en Biotecnología y Licenciatura en Nutrición de la Facultad de Bioquímica y Ciencias Biológicas de la Universidad Nacional del Litoral. http://www.fbcb.unl.edu.ar/pages/estudios/carreras-de-grado.php

12. Food \& Health Survey (2008) (http://www.fbcb.unl.edu.ar/pages/estudios/carreras-de-grado.php)

13. Troncoso C.P. y Amaya J.P.P, 2009. Factores sociales en las conductas alimentarias de estudiantes universitarios. Revista Chilena de nutrición. 36, 4: 1090-1097.

14. Britos S., 2005. Obesidad en Argentina: También una cuestión económica. Buenos Aires, Publicación CESNI. Disponible en: http://www.cesni.org.ar/sistema/archivos/100-obesidad_tambien_una_cuestion_economica.pdf

15. García-García E., Llata-Romero M., Kaufer-Horwitz M. y col., 2008. La obesidad y el síndrome metabólico como problema de salud pública. Una reflexión. Salud pública de México. 50, 6: 530-547.

16. Trescastro-López S., Trescastro-López E.M. y Galiana-Sánchez M.E., 2014. Estudio bibliométrico de programas e intervenciones sobre educación alimentaria y nutricional en el medio escolar en España. Revista Española de Nutrición Humana y Dietética. 18, 4: 184 - 193.

17. Troncoso C., Doepking C. y Silva E., 2011. ¿Es importante la alimentación para los estudiantes universitarios? Medwave. 11, 05: 1-4.

18. Saad Acosta C., Ibáñez E., León C., y col., 2008. Cambios en los hábitos alimentarios en estudiantes de enfermería de la Universidad El Bosque durante su proceso de formación académica, Bogotá, D.C., 2007. Revista Colombiana de Enfermería. 3, 3: 51-60.

19. Cervera Burriel F., Serrano Urrea R., Vico García C. y col., 2013. Hábitos alimentarios y evaluación nutricional en una población universitaria. Nutrición hospitalaria. 28, 2: 438-446.

20. Montero Bravo A. Úbeda M. y García Gonzalez A., 2006. Evaluación de los hábitos alimentarios de una población de estudiantes universitarios en relación con sus conocimientos nutricionales. Nutrición Hospitalaria. 21, 4:466-73.

21. Becerra-Bulla F., Pinzón-Villate G. y Vargas-Zárate M., 2015. Practicas alimentarias de un grupo de estudiantes universitarios y las dificultades percibidas para realizar una alimentación saludable. Revista de la Facultad de Medicina. 63, 3: 457-463.

22. FAO, 2011. La importancia de la educación nutricional. Departamento de agricultura y protección del consumidor. (Consulta: 1/02/2018). Disponible en: http://www.fao.org/nutrition/en/

23. Rodríguez F.R., Palma X.L., Romo A.B y col., 2013. Hábitos alimentarios, actividad física y nivel socioeconómico en estudiantes universitarios de Chile. Nutrición Hospitalaria. 28, 2:447-455. 
24. Rodríguez-Espinoza H., Restrepo-Betancur L.F. y Deossa-Restrepo G.C., 2015. Conocimientos y prácticas sobre alimentación, salud y ejercicio en universitarios de Medellín-Colombia. Perspectivas en Nutrición Humana. 17, 1: 36-54.

25. Intra M.V., Roales-Nieto J.G. y Moreno San Pedro E., 2011. Cambio en las conductas de riesgo y salud en estudiantes universitarios argentinos a lo largo del periodo educativo. International Journal of Psychology and Psychological Therapy. 11, 1: 139-147. 